\title{
Association of serum total bilirubin levels with diastolic dysfunction in heart failure with preserved ejection fraction
}

\author{
Huan Zheng ${ }^{1 \dagger}, \mathrm{Ye} \mathrm{Li}^{2 \dagger}$ and Nanzi Xie ${ }^{1^{*}}$
}

\begin{abstract}
Background: Left ventricular diastolic dysfunction is one of the main characteristics of heart failure patients with a preserved left ventricular ejection fraction. As bilirubin is regarded as an important endogenous antioxidant molecule, serum total bilirubin levels were compared between heart failure patients with a preserved left ventricular ejection fraction and normal controls in this study. We recruited 327 heart failure patients with a preserved left ventricular ejection fraction and 200 healthy controls. Patients were divided into 4 subgroups by their comprehensive echocardiographic manifestations, 1-mild, 2-moderate, 3-severe (reversible restrictive), 4-severe (fixed restrictive). Total bilirubin levels were compared using stepwise multiple regressions adjusted for selected factors.
\end{abstract}

Results: After adjusting for gender, age, smoking, systolic blood pressure, diastolic blood pressure, total cholesterol and triglyceride, serum total bilirubin levels were significantly lower in the heart failure group compared with the control group $(P<0.01)$. Patients in the subgroup (4-severe) showed significantly $(P<0.05)$ lower levels of total bilirubin when compared with the subgroup (1-mild).

Conclusions: TB level was negatively correlated with left ventricular diastolic dysfunction in heart failure patients with a preserved left ventricular ejection fraction, which might provide a new insight into the complicated mechanisms of heart failure with a preserved left ventricular ejection fraction.

Keywords: Bilirubin, Heart failure, Diastolic dysfunction, Echocardiography

\section{Background}

Bilirubin, once considered simply as the metabolic endproduct of heme degradation, has now emerged as an important endogenous antioxidant molecule. Earlier researches have demonstrated an inverse correlation between serum bilirubin and the risk of coronary artery disease and decreased antioxidant activity of bilirubin in atherosclerotic lesions $[1,2]$. Recent studies have confirmed the correlation between serum bilirubin levels and collateral development in patients with chronic total coronary occlusion [3], peripheral arterial disease [4], amputation events in type-2 diabetes mellitus [5], stroke severity and clinical outcomes [6]. Those results suggested that bilirubin may be a part of a cell defense strategy in those macro- and micro- angiopathies.

\footnotetext{
* Correspondence: huanzi318@sohu.com

†Equal contributors

'Geriatrics Department, Tongji Hospital Affiliated to Tongji University, 389

Xincun Road, Shanghai 200065, P.R. China

Full list of author information is available at the end of the article
}

Heart failure with a preserved left ventricular ejection fraction (HFpEF) is a clinical syndrome characterized by the symptoms and signs of heart failure (HF), a preserved left ventricular ejection fraction (LVEF $\geq 50 \%$ ) and abnormal diastolic function [7]. The percentage of patients with HFpEF in epidemiological studies ranges from $40 \%$ to $71 \%$ (mean 56\%), but in hospital-based cohort studies it is slightly lower, ranging from $24 \%$ to $55 \%$ (mean $41 \%$ ) [8]. Despite the better LVEF, HFpEF has a prognosis similar to that observed in HF patients with reduced LVEF, with a mortality rate exceeding $20 \%$ in 1 year [9]. More and more studies have suggested that chronic HF may be potentially characterized as an oxidative disease [10-12]. Recently, Radovanovic et al. showed that by-products of oxidative damage and antioxidant enzyme activities were correlated with echocardiographic indices of remodeling. After a longterm follow-up, they found that the level of malondialdehyde, which was a biomarker of lipid peroxidation, might 
be a useful parameter for monitoring and planning management of chronic HF patients [13].

Diagnostically, echocardiography is an important tool used to identify HFpEF and to stratify impaired diastolic function according to its level of severity (mild, moderate and severe) [14]. Multiple studies revealed a correlation between the severity of diastolic dysfunction and risk of death $[15,16]$. Unlike the association between serum total bilirubin (TB) levels and vascular events, which has been widely studied, whether or not TB plays a role in HFpEF is not yet clear. This study was designed to determine whether TB levels were significantly different in HFpEF patients compared with controls and to investigate the association between TB levels and echocardiographic diastolic dysfunction grades.

\section{Results}

Characteristics of all the HFpEF patients and healthy controls were shown in Table 1 . The 327 HFpEF patients were categorized into four subgroups according to their comprehensive echocardiographic manifestations. The HFpEF group included 130 males and 197 females with a mean age of $66.8 \pm 10.3$ years, and the control group consisted of 116 males and 84 females with a mean age of $43.6 \pm 9.7$ years. In the HFpEF group, $39.8 \%$ of the participants were male and in the control group 58\% were male $(P<0.01)$. Age, body mass index, systolic blood pressure, diastolic blood pressure, total cholesterol, low-density lipoprotein, triglyceride and creatinine levels were all significantly greater in the HFpEF group than in the control group $(P<0.05)$. There were no differences between the two groups with respect to high-density lipoprotein level, fasting glucose, alanine aminotransferase and urea level $(P>0.05)$.

Since significant differences were found between the HFpEF group and the control group for the association of some variables and serum TB levels, differences in TB level between the HFpEF and control group were examined after adjusting for gender, age, smoking, systolic blood pressure, diastolic pressure, total cholesterol and triglyceride. A significantly lower serum level of TB was found in the HFpEF group compared with the control group after adjustment $(P<0.01)$. In addition, the level of TB was significantly lower in patients with grade 4 than those with grade 1 disease $(P<0.05)$.

Simple linear regression and multivariate regression with stepwise selection were used to evaluate factors associated with TB level (Table 2). During the stepwise selection process, we adopted the approach of bidirectional elimination, testing at each step for variables to be included or excluded $\left(\alpha_{\text {in }}=0.05, \alpha_{\text {out }}=0.10\right)$. Results showed that gender, age and diastolic dysfunction echocardiographic grading were significantly associated with TB level $\left(R^{2}=\right.$ 0. 261, $P<0.05)$.

\section{Discussion}

In this study, none of the HFpEF patients had any relevant diseases that could reduce the serum TB level, and we found that serum TB levels were significantly lower in HFpEF patients than those in normal controls after adjusting for age, gender, body mass index, smoking, systolic blood pressure, diastolic blood pressure, total cholesterol and triglyceride. In addition, the serum TB levels in HFpEF grade 4 (severe, fixed restrictive) was significantly lower than those in $\mathrm{HFpEF}$ grade 1 (mild). Last but not least, HFpEF echocardiophic grading was significantly associated with serum TB levels in simple linear regression and multivariate regression analysis.

\section{Pathophysiology of HFpEF}

Recent epidemiological studies have provided growing recognition that HFpEF is a common and costly clinical entity that is increasing in prevalence $[8,9]$. The pathophysiology of HFpEF is distinct from that of HF with reduced LVEF and is characterized by left ventricular (LV) diastolic dysfunction [17]. Echocardiography is an essential non-invasive tool useful for diagnosing and classifying LV diastolic dysfunction. A key component of LV diastolic dysfunction is LV stiffness causing inability to dilate sufficiently to cope with the venous supply from the lungs. This stiffness can be increased by any pathological process affecting the heart muscle, the most common causes being LV hypertrophy and/or myocardial ischemic lesions [18]. In addition, stiffness is aggravated by changes in extracellular matrix collagen deposition, and this can even precede LV hypertrophy [19]. Meanwhile, it has been pointed out that free radicals can be involved in cardiomyocyte hypertrophy, apoptosis and mechanisms of cardiac remodeling [10,11]. Giordano supported the concept of reactive oxygen species contributing to the cardiac remodeling processes in a number of ways, including activating matrix metalloproteinases that participate in reconfiguration of the extracellular matrix, acting as signaling molecules in the development of compensatory hypertrophy and myocyte loss via apoptosis or other cell death mechanisms. The role of oxidative stress in LV diastolic dysfunction is very complex and those concrete pathways are still unclear; however, existence of systemic oxidative stress in chronic HF and its gradual increase as chronic HF progresses have been confirmed [13]. In recent years, the improved treatment strategy has increased survival rate in systolic HF, whereas the prognosis for HFpEF remains unchanged [9]. Risk stratification is an integral part of management in patients with HFpEF, and is also an important step in defining the optimal treatment strategy. This is why researches are searching for new biomarkers to help probe the complicated pathophysiology of HFpEF.

\section{Antioxidant effects of TB}

Since the report in 1994 by Schwertner et al. which indicated that serum bilirubin level was negatively correlated 
Table 1 Characteristics of the controls and HFpEF patients

\begin{tabular}{|c|c|c|c|c|c|c|}
\hline & \multirow{2}{*}{$\begin{array}{l}\text { Control group } \\
(n=200)\end{array}$} & \multicolumn{5}{|c|}{ HFpEF groups } \\
\hline & & $\begin{array}{l}\text { 1-mild } \\
(n=52)\end{array}$ & $\begin{array}{l}\text { 2-moderate } \\
(n=116)\end{array}$ & $\begin{array}{l}\text { 3-severe (reversible } \\
\text { restrictive) }(n=138)\end{array}$ & $\begin{array}{l}\text { 4-severe (fixed } \\
\text { restrictive) }(n=21)\end{array}$ & $\overline{P^{*} \text {-value }}$ \\
\hline Male (n, \%) & $116(58 \%)$ & $31(5.6 \%)^{\mathrm{a}}$ & $44(37.9 \%)^{a}$ & $49(35.5 \%)^{a}$ & $6(28.6 \%)^{a, b, c, c, d}$ & 0.003 \\
\hline Age (years) & $43.6(9.7)$ & $69.7(7.3)^{\mathrm{a}}$ & $65.9(10.2)^{\mathrm{a}}$ & $67.1(11.4)^{\mathrm{a}}$ & $66.3(13.1)^{a}$ & 0.001 \\
\hline $\mathrm{BMI}\left(\mathrm{kg} / \mathrm{m}^{2}\right)$ & $22.7(2.5)$ & $24.3(3.6)^{\mathrm{a}}$ & $23.9(4.7)$ & $24.5(2.1)^{\mathrm{a}}$ & $23.8(3.7)$ & 0.048 \\
\hline Smoker (n, \%) & $85(42.5 \%)$ & $25(48.1 \%)$ & $39(33.6 \%)$ & $38(27.5 \%)^{a}$ & $5(23.8 \%)^{a, b, c}$ & 0.028 \\
\hline \multicolumn{7}{|l|}{ Medications } \\
\hline ASA $(n, \%)$ & 0 & $48(92.3 \%)^{\mathrm{a}}$ & $110(94.8 \%)^{\mathrm{a}}$ & $134(97.1 \%)^{\mathrm{a}}$ & $20(95.2 \%)^{a}$ & $<0.001$ \\
\hline Diuretic (n, \%) & 0 & $21(40.4)^{a}$ & $64(55.2 \%)^{a, b}$ & $70(50.7 \%)^{a, b}$ & $18(85.7 \%)^{a, b c, d}$ & $<0.001$ \\
\hline Beta blocker (n, \%) & 0 & $21(40.4 \%)^{a}$ & $44(37.9 \%)^{a}$ & $50(36.2 \%)^{a}$ & $8(38.1 \%)^{a}$ & $<0.001$ \\
\hline ACEI/ARB (n, \%) & 0 & $47(90.4 \%)^{\mathrm{a}}$ & $112(96.6 \%)^{a}$ & $133(96.4 \%)^{\mathrm{a}}$ & $21(100 \%)^{a}$ & $<0.001$ \\
\hline Statin $(n, \%)$ & 0 & $50(96.2 \%)^{a}$ & $111(95.7 \%)^{a}$ & $132(95.7 \%)^{a}$ & $19(90.5 \%)^{a}$ & $<0.001$ \\
\hline CCB $(n, \%)$ & 0 & $27(51.9 \%)^{a}$ & $52(44.8 \%)^{a}$ & $66(47.8 \%)^{\mathrm{a}}$ & $9(42.9 \%)^{\mathrm{a}}$ & $<0.001$ \\
\hline $\mathrm{HR}(\mathrm{b} / \mathrm{min})$ & $77.4(6.1)$ & $75.8(6.7)$ & $78.2(7.1)$ & $71.1(5.8)^{\mathrm{a}}$ & $80.2(7.2)^{d}$ & 0.036 \\
\hline $\mathrm{SBP}(\mathrm{mmHg})$ & $120.1(8.6)$ & $124.6(5.8)$ & $138.1(10.1)^{a, b}$ & $140.2(9.3)^{a, b}$ & $150.6(7.2)^{a, b, c}$ & 0.005 \\
\hline $\mathrm{DBP}(\mathrm{mmHg})$ & $78.2(7.0)$ & $80.3(8.8)$ & $81.2(6.5)$ & $90.2(10.3)^{a, b, c}$ & $91.4(9.5)^{a, b, c}$ & 0.017 \\
\hline Total cholesterol (mg/dl) & $191.7(19.1)$ & $209.3(20.2)^{\mathrm{a}}$ & $234.7(19.0)^{a}$ & $225.6(18.8)^{a}$ & $242.5(22.7)^{a}$ & 0.007 \\
\hline $\mathrm{LDL}(\mathrm{mg} / \mathrm{dl})$ & $108.3(17.4)$ & $124.1(18.2)^{\mathrm{a}}$ & $120.6(19.1)^{\mathrm{a}}$ & $125.7(18.8)^{\mathrm{a}}$ & $131.9(21.2)^{\mathrm{a}}$ & 0.004 \\
\hline $\mathrm{HDL}(\mathrm{mg} / \mathrm{dl})$ & $51.3(7.5)$ & $43.4(8.2)$ & $44.0(8.1)$ & $47.9(6.3)$ & $45.2(7.7)$ & 0.069 \\
\hline Triglyceride (mg/dl) & $128.8(19.4)$ & $150.9(17.3)^{\mathrm{a}}$ & $152.6(18.4)^{\mathrm{a}}$ & $147.6(20.5)^{\mathrm{a}}$ & $144.1(19.6)^{\mathrm{a}}$ & 0.025 \\
\hline Fasting glucose (mg/dl) & $95.2(7.3)$ & $96.3(8.1)$ & $100.7(6.8)$ & $94.9(7.4)$ & $102.5(8.2)$ & 0.326 \\
\hline Creatinine (umol/L) & $83.6(10.7)$ & $92.4(11.1)$ & $95.3(9.6)$ & $121.3(12.5)^{a, b, c}$ & $127.6(13.5)^{a, b, c}$ & 0.013 \\
\hline Urea level (umol/L) & $261.4(23.9)$ & $252.4(22.1)$ & $274.5(19.3)$ & $282.6(20.7)$ & $288.2(21.6)$ & 0.204 \\
\hline Alanine aminotransferase $(\mathrm{U} / \mathrm{L})$ & $25.4(6.4)$ & $32.1(7.9)$ & $29.6(8.1)$ & $30.1(6.4)$ & $32.1(7.5)$ & 0.338 \\
\hline TB (umol/L) & $15.3(4.1)$ & $14.1(3.2)$ & $12.5(3.6)^{\mathrm{a}}$ & $11.8(2.9)^{a}$ & $10.4(4.0)^{a, b}$ & 0.001 \\
\hline
\end{tabular}

Values are mean \pm SD or numbers. BMI, body mass index; HR, heart rate; ASA: acetylsalicylic acid; ACEl: angiotensin-converting enzyme inhibitor; ARB: angiotensin II receptor blocker; CCB: calcium channel blocker; SBP, systolic blood pressure; DBP, diastolic blood pressure; LDL, low density lipoprotein; HDL, high density lipoprotein; TB, total bilirubin; Numerical variables were compared by $t$-test; Categorical variables (male/female, smoker, medications) were compared by chi-square test. $P^{*}$-value, control group vs. HFpEF group; ${ }^{a} P<0.05$ compared with control, after Bonferroni correction; ${ }^{b} P<0.05$ compared with stage 1-mild, after Bonferroni correction; ${ }^{c} P<0.05$ compared with stage 2-modeate, after Bonferroni correction; ${ }^{\mathrm{d}} P<0.05$ compared with stage 3 -severe (reversible restrictive), after Bonferroni correction.

with coronary artery disease, many studies have observed that in the general population, upper normal or modestly elevated bilirubin levels are associated with improved outcomes, such as a lower risk of coronary heart disease events, respiratory disease, and all-cause mortality [20-22]. A previous study showed that serum bilirubin levels were inversely correlated with the superoxide anion radicals generated by mononuclear cells in cardiac syndrome $\mathrm{X}$ patients [23]. In 2012, a study on a large statin-treated cohort reported that serum bilirubin level measured before a statin prescription to assess liver function was an independent risk factor for cardiovascular disease and death in both men and women [21]. It showed that the incidence of cardiovascular disease in the lowest decile category of bilirubin was 215 per 10,000 person-years compared with 163 per 10,000 person-years in the highest decile. As for the relationship between bilirubin and HF, the conclusions are still controversial. For example, a recent analysis of the Efficacy of Vasopressin Antagonism in Heart Failure Outcome Study with Tolvaptan (EVEREST) cohort, which included patients hospitalized with systolic HF, showed that an elevated baseline TB level and inhospital increase in bilirubin were associated with an increased risk of all-cause mortality and combined cardiovascular mortality [24]. While in another study which focused on the relationship between liver function abnormalities and hemodynamic profile, it turned out that the predictors of all-cause mortality were $\gamma$-glutamyl transpeptidase, alkaline phosphatase, aspartate aminotransferase and lactic dehydrogenase, other than TB [25]. However, there were fewer data investigating the relationship between TB and diastolic dysfunction. In our study, serum TB level in HFpEF was lower than that in the normal controls, and serum TB level in grade subgroup 4 was lower than that in grade subgroup 1 . To our knowledge, this is the first study to investigate the association between serum TB level and 
Table 2 Results of univariate and multivariate regression with stepwise selection estimating TB levels

\begin{tabular}{|c|c|c|c|c|c|c|}
\hline \multirow[t]{2}{*}{ Varibles } & \multicolumn{3}{|c|}{ Univariate } & \multicolumn{3}{|c|}{ Multivariate } \\
\hline & $\beta$ & $\mathrm{SE}(\beta)$ & $P$ & $\beta$ & $\mathrm{SE}(\beta)$ & $P$ \\
\hline Male & 1.62 & 0.32 & 0.002 & 3.47 & 0.83 & 0.001 \\
\hline Age & 0.06 & 0.02 & 0.021 & 0.09 & 0.04 & 0.003 \\
\hline BMI & 0.24 & 0.06 & 0.375 & & & \\
\hline Smoke & -0.57 & 0.39 & 0.427 & & & \\
\hline Total cholesterol & -0.38 & 0.14 & 0.224 & & & \\
\hline HDL cholesterol & 0.69 & 0.41 & 0.573 & & & \\
\hline LDL cholesterol & -0.51 & 0.10 & 0.106 & & & \\
\hline Triglyceride & 0.82 & 0.37 & 0.483 & & & \\
\hline SBP & -0.12 & 0.06 & 0.504 & & & \\
\hline DBP & -0.08 & 0.14 & 0.669 & & & \\
\hline Diastolic dysfunction grade & -0.92 & 0.35 & 0.037 & -1.03 & 0.39 & 0.021 \\
\hline
\end{tabular}

BMI, body mass index; HDL, high density lipoprotein; LDL, low density lipoprotein; SBP, systolic blood pressure; DBP, diastolic blood pressure; $\beta$, standardized regression coefficient; $\mathrm{SE}(\beta)$, standard error of the standardized regression coefficient. Correlation between the continuous variable and TB level was made by Pearson's method; correlation between the discrete variable and TB level was made by Spearman's method; Multivariate regression model: $a_{\text {in }}=0.05, a_{\text {out }}=0.10, R^{2}=0.261$.

diastolic dysfunction grading in HFpEF. We postulated those results might be related to the antioxidant effects of bilirubin. As we all know, HF may cause hepatic congestion and reduced forward flow, which would lead to elevated TB levels, but we found that it was negatively correlated with the severity of our patients' LV diastolic dysfunction. This finding implied that some potential benefits might outweigh the underlying severity of HF causing the TB level increasing. The mechanisms linking low bilirubin and risk or worse outcomes for cardiovascular disease are not fully understood. A number of mechanisms has been proposed for the antioxidant effects of bilirubin, including suppression of low-density lipoprotein oxidation [26], monocyte migration [27], vascular smooth muscle cell proliferation [28] and endothelial dysfunction [29]. Early animal experiments indicated that bilirubin served as a physiological antioxidant in ischemia-reperfusion [30] and served a protective function against injury-mediated proliferation of intimal cells [31]. It was reported that moderate hyperbilirubinemia reduced blood pressure in angiotensin II-dependent hypertension through decreasing oxidative stress and increasing nitric oxide levels [32]. Our study was only a clinical observation, further experiments are needed to explore the underlying mechanisms.

\section{Biomarkers of HFpEF}

The best characterized biomarkers in patients with HFpEF are the natriuretic peptides: B-type natriuretic peptide (BNP) and N-terminal pro-BNP (NT pro-BNP) [29]. BNP is mainly released in response to increased cardiac volume and pressure overload. It is synthesized as an inactive pro- hormone that is split into the active hormone BNP and the inactive NT pro-BNP. Both elevated NT pro-BNP and $\mathrm{BNP}$ are strong independent predictors of clinical events in patients with HFpEF. No consensus exists on use of natriuretic peptide levels to guide medical therapy [33]. Our study was an attempt to invesigate whether bilirubin might play an role in the pathogenic processes that link oxidative stress and diastolic dysfunction in HFpEF patients.

\section{Limitations}

There were potential limitations to this study. First, the sample size was relatively small. Further studies with larger sample sizes are needed to confirm our results. Second, our study was cross-sectional, and it would have been ideal to have obtained repeated determinations in the same patients over time in our study to prove their relationship.

\section{Conclusions}

The results of this study indicated a correlation between decreased serum TB level and increasing severity of LV diastolic dysfunction in HFpEF. Further in vivo studies investigating the mechanisms underlying decreased TB in patients with HFpEF may assist in developing new targets for prevention and treatment.

\section{Methods \\ Subjects}

This observational study included 327 patients (130 male and 197 female, aging $66.8 \pm 10.3$ years old) with $\mathrm{HFpEF}$ who were hospitalized in our department during the period from January 2008 to January 2013 and a normal control group consisting of 200 healthy volunteers (116 male and 84 female, aging $43.6 \pm 9.7$ years old) who attended an annual checkup at our hospital in 2012. HFpEF was diagnosed according to criteria in the consensus statement of the Heart Failure and Echocardiography Associations of the European Society of Cardiology [7]. Patients with systolic HF, severe valvular stenosis or regurgitation, congenital heart disease, atrial fibrillation, chronic obstructive pulmonary disease, malignancy, other extracellular fluid-increasing diseases such as hypothyroidism, diabetes, serious cerebrovascular diseases, a history of liver or gallbladder or bile-duct disease, hemolytic disease, rheumatic or immune diseases were excluded. The control participants had no cardiovascular or any other organ system disease, and had normal physical examination, chest roentgenogram, electrocardiogram, two-dimensional and Doppler echocardiogram. All participants gave their informed consent, and the study protocol was approved by ethical review board of Tongji Hospital Affilated to Tongji University, Shanghai, China. 


\section{Laboratory measurement}

$5 \mathrm{ml}$ venous blood sample was collected from each participant under fasting conditions. Then they were centrifuged and the serum was extracted for use, with spinning speed $800 \mathrm{~g}$ for 5 mins at $4^{\circ} \mathrm{C}$ and storage temperature $-80^{\circ} \mathrm{C}$. Fasting blood glucose, total cholesterol, high-density lipoprotein cholesterol, low-density lipoprotein cholesterol, triglyceride, uric acid, creatinine and TB levels were recorded. TB levels were analyzed using an autoanalyzer (Roche Diagnostic Modular Systems, Tokyo, Japan).

\section{Echocardiography}

All participants underwent complete transthoracic echocardiographic studies (Vivid 7 system, General Motor Corp., USA) using a 2.5-4.0-MHz transducer. LVEF was measured using Simpson's method. The following conventional mitral inflow pulse wave Doppler parameters were measured: peak early (E) and late diastolic (A) transmitral filling flow velocities, E/A ratio, $\Delta \mathrm{E} / \mathrm{A}$ with a Valsalva maneuver and deceleration time (DT) of the $\mathrm{E}$ wave and isovolumic relaxation time (IRT), defined as the time from aortic valve closure to mitral valve opening. Pulmonary venous flow parameters were also measured: peak systolic velocity (Ps), peak diastolic velocity (Pd), Ps/Pd ratio, atrial reversal (PVAr) velocity, the difference in PVAr and transmitral A velocity duration (PVAr-A). Tissue Doppler parameters were measured: peak systolic mitral annular velocity $\left(S^{\prime}\right)$, early diastolic mitral annular velocity (E') and late diastolic mitral velocity $\left(\mathrm{A}^{\prime}\right)$. Those parameters were obtained from the apical four-chamber view, 1-3-mm sample volume for mitral leaflets, $2-3-\mathrm{mm}$ for pulmonary vein, $2-5-\mathrm{mm}$ for mitral leaflets, respectively. The mean of three measurements was used for analysis of the Doppler data. The ratio of mitral peak velocity of early diastolic filling to early diastolic mitral annular velocity (E/E') was calculated for the lateral and septal annulus. All the HFPEF patients were divided into four groups: 1 - mild, 2 - moderate, 3 - severe (reversible restrictive), $4-$ severe (fixed restrictive) [14]. 1 (mild): $\mathrm{E} / \mathrm{A}<0.8, \Delta \mathrm{E} / \mathrm{A}$ (Valsalva maneuver) $\langle 0.5$, DT $\rangle$ $200 \mathrm{~ms}$, E/E'lateral $<8$, Ps $>$ Pd, PVAr-A $<30$ ms; 2 (moderate): $0.8<\mathrm{E} / \mathrm{A}<2, \Delta \mathrm{E} / \mathrm{A}$ (Valsalva maneuver) $\geq 0.5,160<$ DT $<200 \mathrm{~ms}$, E/E'lateral $\geq 8-12$, Ps $<$ Pd, PVAr-A $\geq 30$ ms; 3 (severe) (reversible restrictive): $\mathrm{E} / \mathrm{A} \geq 2, \Delta \mathrm{E} / \mathrm{A}$ (Valsalva maneuver) $\geq 0.5$, DT $<160 \mathrm{~ms}$, E/ E'lateral $>12$, $\mathrm{Ps}<\mathrm{Pd}$, PVAr$\mathrm{A} \geq 30 \mathrm{~ms} ; 4$ (severe) (fixed restrictive): $\mathrm{E} / \mathrm{A} \geq 2, \Delta \mathrm{E} / \mathrm{A}$ (Valsalva maneuver) $<0.5$, DT $<160 \mathrm{~ms}$, E/ E'lateral $>12$, $\mathrm{Ps}<\mathrm{Pd}$, PVAr-A $\geq 30 \mathrm{~ms}$.

\section{Statistical analysis}

All statistical analyses were carried out using statistical software (SPSS, version 15.0 for Windows; SPSS Inc., USA). All numeric variables were expressed as mean $\pm \mathrm{SD}$ and normally distributed. We performed a hierarchal analysis between two groups (controls and $\mathrm{HFpEF}$ ) and then a further analysis among 5 groups (Control, 1-mild, 2-moderate, 3severe reversible, 4-severe fixed) with Bonferroni-correction. Numerical variables were compared by $t$-test and categorical variables were compared by chi-square test. Simple linear regression and multivariate regression with stepwise selection were used to evaluate factors associated with TB levels. Probability values of $P<0.05$ were considered significant.

\section{Competing interests}

The authors declare that they have no competing interests.

\section{Authors' contributions}

Huan Zheng collected the clinical data; Ye Li was responsible for the data analysis;Nanzi Xie designed the study and editing the manuscript writing. All authors read and approved the final manuscript.

\section{Author details}

${ }^{1}$ Geriatrics Department, Tongji Hospital Affiliated to Tongji University, 389 Xincun Road, Shanghai 200065, P.R. China. ${ }^{2}$ Heart Failure Department, Shanghai East Hospital Affiliated to Tongji university, Shanghai, China.

Received: 22 July 2013 Accepted: 18 November 2013

Published: 26 March 2014

\section{References}

1. Schwertner HA, Jackson WG, Tolan G: Association of low serum concentration of bilirubin with risk of coronary artery disease. Clin Chem 1994, 40:18-23.

2. Kawamura K, Ishikawa K, Wada Y, Kimura S, Matsumoto H, Kohro T, Itabe $H$, Kodama T, Maruyama Y: Bilirubin from heme oxygenase-1 attenuates vascular endothelial activation and dysfunction. Arterioscler Thromb Vasc Biol 2005, 25:155-160.

3. Erdogan $T$, Ciçek $Y$, Kocaman SA, Canga A, Cetin M, Durakoglugil E, Satiroglu O, Temiz A, Ergül E, Sahin I, Bostan M: Increased serum bilirubin level is related to good collateral development in patients with chronic total coronaryocclusion. Intern Med 2012, 51:249-255.

4. Wang HY, Han P, Zhang WH, Liu B, Li HL, Wang HJ, Huang RP: Serum bilirubin level is negatively correlated with disease progression of peripheral arterial disease: an observational cohort study. Angiology 2012, 63:248-253.

5. Chan KH, O'Connell RL, Sullivan DR, Hoffmann LS, Rajamani K, Whiting M, Donoghoe MW, Vanhala M, Hamer A, Yu B, Stocker R, Ng MK, Keech AC, FIELD Study Investigators: Plasma total bilirubin levels predict amputation events in type 2 diabetes mellitus: the Fenofibrate Intervention and Event Lowering in Diabetes (FIELD) study. Diabetologia 2013, 56:724-736.

6. Xu T, Zhang J, Xu T, Liu W, Kong Y, Zhang Y: Association of serum bilirubin with stroke severity and clinical outcomes. Can J Neurol Sci 2013, 40:80-84.

7. Paulus WJ, Tschöpe C, Sanderson JE, Rusconi C, Flachskampf FA, Rademakers FE, Marino P, Smiseth OA, De Keulenaer G, Leite-Moreira AF, Borbély A, Edes I, Handoko ML, Heymans S, Pezzali N, Pieske B, Dickstein K, Fraser AG, Brutsaert DL: How to diagnose diastolic heart failure: a consensus statement on the diagnosis of heart failure with normal left ventricular ejection fraction by the Heart Failure and Echocardiography Associations of the European Society of Cardiology. Eur Heart J 2007, 28:2539-2550.

8. Hogg K, Swedberg K, McMurray J: Heart failure with preserved left ventricular systolic function; epidemiology, clinical characteristics, and prognosis. J Am Coll Cardiol 2004, 43:317-327.

9. Bhatia RS, Tu JV, Lee DS, Austin PC, Fang J, Haouzi A, Gong Y, Liu PP: Outcome of heart failure with preserved ejection fraction in a population-based study. N Engl J Med 2006, 355:260-269.

10. Valgimigli M, Merli E, Malagutti P, Soukhomovskaia O, Cicchitelli G, Antelli A, Canistro D, Francolini G, Macrì G, Mastrorilli F, Paolini M, Ferrari R: Hydroxyl radical generation, levels of tumor necrosis factor-alpha, and progression to heart failure after acute myocardial infarction. J Am Coll Cardiol 2004, 43:2000-2008

11. Giordano FJ: Oxygen, oxidative stress, hypoxia and heart failure. J Clin Invest 2005, 115:500-508. 
12. Seddon M, Looi YH, Shah AM: Oxidative stress and redox signaling in cardiac hypertrophy and heart failure. Heart 2007, 93:903-907.

13. Radovanovic S, Savic-Radojevic A, Pljesa-Ercegovac M, Djukic T, Suvakov S, Krotin M, Simic DV, Matic M, Radojicic Z, Pekmezovic T, Simic T: Markers of oxidative damage and antioxidant enzyme activities as predictors of morbidity and mortality in patients with chronic heart failure. $J$ Card Fail 2012, 18:493-501.

14. Nagueh SF, Appleton CP, Gillebert TC, Marino PN, Oh JK, Smiseth OA, Waggoner AD, Flachskampf FA, Pellikka PA, Evangelisa A: Recommendations for the evaluation of left ventricular diastolic function by echocardiography. J Am Soc Echocardiogr 2009, 22:107-133.

15. Achong N, Wahi S, Marwick TH: Evolution and outcome of diastolic dysfunction. Heart 2009, 95:813-818.

16. Bella JN, Palmieri V, Roman MJ, Liu JE, Welty TK, Lee ET, Fabsitz RR, Howard BV, Devereux RB: Mitral ratio of peak early to late diastolic filling velocity as a predictor of mortality in middle-aged and elderly adults: the strong heart study. Circulation 2002, 105:1928-1933.

17. Zile MR, Baicu CF, Gaasch WH: Diastolic heart failure-abnormalities in active relaxation and passive stiffness of the left ventricle. $N$ Engl J Med 2004, 350:1953-1959.

18. Grossman W, Barry WH: Diastolic pressure-volume relations in the diseased heart. Fed Proc 1980, 39:148-155.

19. Borbély A, Papp Z, Edes I, Paulus WJ: Molecular determinants of heart failure with normal left ventricular ejection fraction. Pharmacol Rep 2009, 61:139-145.

20. Hunt SC, Kronenberg F, Eckfeldt JH, Hopkins PN, Myers RH, Heiss G: Association of plasma bilirubin with coronary heart disease and segregation of bilirubin as a major gene trait: the NHLBI family heart study. Atherosclerosis 2001, 154:747-754.

21. Horsfall $L$, Nazareth I, Petersen I: Cardiovascular events as a function of serum bilirubin levels in a large, statin-treated cohort. Circulation 2012, 126:2556-2564.

22. Temme EH, Zhang J, Schouten EG, Kesteloot H: Serum bilirubin and 10year mortality risk in a Belgian population. Canc Causes Contr 2001, 12:887-894

23. Huang SS, Huang PH, Leu HB, Wu TC, Lin SJ, Chen JW: Serum bilirubin predicts long-term clinical outcomes in patients with cardiac syndrome X. Heart 2010, 96:1227-1232.

24. Ambrosy AP, Vaduganathan M, Huffman MD, Khan S, Kwasny MJ, Fought AJ, Maggioni AP, Swedberg K, Konstam MA, Zannad F, Gheorghiade M: EVEREST trial investigators: Clinical course and predictive value of liver function tests in patients hospitalized for worsening heart failure with reduced ejection fraction: an analysis of the EVEREST trial. Eur I Heart Fail 2012, 14:302-311.

25. van Deursen VM, Damman K, Hillege HL, van Beek AP, van Veldhuisen DJ, Voors AA: Abnormal liver function in relation to hemodynamic profile in heart failure patients. J Card Fail 2010, 16:84-90

26. Neuzil J, Stocker R: Free and albumin-bound bilirubin are efficient coantioxidants for alpha-tocopherol, inhibiting plasma and low density lipoprotein lipid peroxidation. J Bio IChem 1994, 269:16712-16719.

27. Ishikawa K, Navab M, Leitinger N, Fogelman AM, Lusis AJ: Induction of hemeoxygenase-1 inhibits the monocyte transmigration induced by mildly oxidized LDL. J Clin Invest 1997, 100:1209-1216.

28. Ollinger R, Bilban M, Erat A, Froio A, McDaid J, Tyagi S, Csizmadia E, GraçaSouza AV, Liloia A, Soares MP, Otterbein LE, Usheva A, Yamashita K, Bach FH: Bilirubin: a natural inhibitor of vascular smooth muscle cell proliferation. Circulation 2005, 112:1030-1039.

29. Erdogan D, Gullu H, Yildirim E, Tok D, Kirbas I, Ciftci O, Baycan ST, Muderrisoglu H: Low serum bilirubin levels are independently and inversely related to impaired flow-mediated vasodilation and increased carotid intima-media thickness in both men and women. Atherosclerosis 2006, 184:431-437.

30. Yamaguchi T, TerakadoM HF, Aoki K, TanakaM NH: Role of bilirubin as antioxidant in an ischemia-reperfusion of rat liver and induction of hemeoxygenase. Biochem Biophys Res Commun 1996, 223:129-135.
31. Aizawa T, Ishizaka N, Taguchi J, Kimura S, Kurokawa K, Ohno M: Balloon injury does not induce heme oxygenase- 1 expression, but administration of hemininhibits neointimal formation in balloon-injured rat carotid artery. Biochem Biophys Res Commun 1999, 261:302-307.

32. Vera T, Stec DE: Moderate hyperbilirubinemia improves renal hemodynamics in ANG II-dependent hypertension. Am J Physiol Regul Integr Comp Physiol 2010, 299:R1044-R1049.

33. Meyer T, Shih J, Aurigemma G: Heart failure with preserved ejection fraction (diastolic dysfunction). Ann Intern Med 2013, 158:ITC5-1-ITC5-15.

doi:10.1186/0717-6287-47-7

Cite this article as: Zheng et al:: Association of serum total bilirubin levels with diastolic dysfunction in heart failure with preserved ejection fraction. Biological Research 2014 47:7.

\section{Submit your next manuscript to BioMed Central and take full advantage of:}

- Convenient online submission

- Thorough peer review

- No space constraints or color figure charges

- Immediate publication on acceptance

- Inclusion in PubMed, CAS, Scopus and Google Scholar

- Research which is freely available for redistribution

Submit your manuscript at www.biomedcentral.com/submit
Ciomed Central 